\title{
A New Kind of Transformer Oil State Detection Method based on Multi-frequency Detection Technique and Multivariate Statistics
}

\author{
Rui Rao, a , Zhengjia Li ${ }^{2, b}$, Haoyong Song ${ }^{1, c}$, Yuqing Chen ${ }^{1, d}$, and Dan $\mathrm{Li}^{1, \mathrm{e}}$ \\ ${ }^{1}$ Electric Power Research Institute of Guangzhou Power Supply Bureau Co.,Ltd, Guangzhou, \\ China \\ ${ }^{2}$ Huatian Power Technology Co., Ltd, Suzhou, China \\ apowertoyou@163.com, bzjli@huatianpower.com, shy_gz@163.com, dcyq_gz@163.com, \\ 'Idan_gz@163.com
}

\begin{abstract}
Keywords: multi-frequency ultrasound; transformer oil; fault detection; multivariate statistical analysis; complex artificial neural network; self-learning

Abstract. The operating status of transformers directly affects the safety of entire power grid. Therefore, it is essential to monitor a variety of transformer fault and ensure the normal operation of power grid. The multi-frequency ultrasonic technique was proposed in the thesis and which utilized hundreds of different frequency ultrasonic signals to test transformer oil for transformer fault. Various ultrasonic parameters are received and based on which the multivariate statistical analysis method and the plural artificial neural network data analysis method are applied to obtain the characteristic values, and the operation state values are compared with the standard values stored in the background database, so that the evaluations can be made on transformer's current operation situations. The design principle of the transformer oil measurement system was elaborated and a multivariate statistics method was given in the paper and which was applied to build a certain internal relationship between the ultrasonic parameters and stored sample values in database, and then the state of transformer that contained in the ultrasonic parameters can be released. An experimental test verifies that the developed system can effectively and accurately detect multiple transformer faults via detailed analysis of ultrasonic parameters of transformer oil, thus provides significant guidance and reference for the transformer detection departments.
\end{abstract}

\section{Introduction}

The electric equipment, as core parts of smart grid, whose normal operation is fundamental assurance of power grid safety. Among them, the transformers are key equipment, and are also a kind of large numbers of operating equipment in grid. It's necessary to find the faults may take place timely along with the transformer conditions, and which has been a significant approach to guarantee power supply reliability ${ }^{[1-3]}$.

The conventional detection method is only singly implemented to measure after taking sample, which cannot reflect the true condition of transformer oil during transformer operation. The water in transformer insulation paper is transferred to the oil due to changes of temperature, resulting in extreme variation of breakdown voltage values. In order to better observe this variation process, it's required to establish a transformer mode and temperature calculation model with same proportion in laboratory, and utilize the system software to analyze and compute the modes established, and on the basis, the impacts of dynamic transferring process of water on transformer insulation oil parameters in different temperature can be analyzed truly.

Currently, the techniques applied in transformer detection are mainly oil chromatographic analysis, infrared temperature measurement and partial discharge online detection, etc. For example, adopt the oil chromatographic analysis technique to perform analysis of faults exist in transformers, while use the gas chromatography method to detect dissolved gas in oil and during the whole detection process from taking oil samples to oil gas separation and then to chromatographic analysis, many disadvantages of many links, cockamamie operation procedures, and long test period, etc., so that, inevitably, the larger 
test errors were introduced ${ }^{[1,4]}$, this detection method was not timely enough to detect the faults with rapid variation, and was difficult to play its role.

The literature ${ }^{[5,6]}$ proposed infrared temperature measurement technique for transformer detection, which had advantages of non-contact measurement, wide measurement range and fast temperature measurement, while it was easily influenced by environment factors, and for light or polished metal surface, the influences of temperature readings were large, and could only measure the external temperature of transformer, and couldn't measure the temperature inside transformer and when the faults existed, and the temperature was just one of transformer parameters, thus couldn't reflect the operation state of transformer according to the temperature.

The literature ${ }^{[7-9]}$ adopted partial discharge test to detect transformer faults, and for this method, due to weak partial discharge signal, the field disturbances were strong, and sometimes the disturbance signals were stronger than 2 to 3 times of partial discharge signals, so that it couldn't distinguish partial discharge signals accurately and restrain interferences effectively, and the measured results had a larger error, which was a larger limitation of partial discharge online detection technique.

The thesis implemented research on transformer detection method based on multi-frequency ultrasonic principle, in which, several hundreds of ultrasonic parameters could be gathered in a measurement scan frequency in a second. Then the ultrasonic receiving device performed detection and analysis on the reflective ultrasonic signals, and which contained the concrete features information of different materials in transformer oil. The detection data analysis was made on the large amount of testing data, and multi transformer oil parameters could be obtained which included micro water content, breakdown voltage, dielectric loss factor, oil conductivity and oil surface tension, etc. further, the data analysis software was designed based on the multivariate statistical analysis technique and complex artificial neural network, then a database contained various types of oil samples was established. A series of smart algorithms are used to build relationship between these characteristic values (ultrasonic parameters) and oil sample parameters, through the analysis of transformer oil parameters the true operation state of transformer could be obtained. Therefore, the thesis has mainly done the following work .

1) Proposed that utilized multi-frequency ultrasonic technique for transformer detection;

2) Developed single device which could measure multi kinds of transformer oil parameters, and which included the moisture water content, breakdown voltage, dielectric loss factor, oil conductivity and oil surface tension, etc.

3) Achieved the breakdown voltage and dielectric loss factors detection of transformer in conditions of without power outage and high pressure;

4) Simplified the conventional transformer detection and operation methods, and timely and totally understand operation state information of transformers.

\section{Algorithm Study}

Develop the data analysis software based on multivariate statistics analysis [10] and complex artificial neural network and which is used to make analysis and processing on the collected multi frequency ultrasonic parameters, including ultrasonic amplitudes, phase, frequency, and flight time, so as to judge concrete fault names of transformer.

Mathematical Model of Multivariate Statistics Analysis. Multivariate statistics analysis is an effective method to deal with the multidimensional or multi variables data, eliminate some redundant and invalid data, thus achieve the purpose of dimension reduction ${ }^{[11,12]}$, then according to the limited linear uncorrelated data, the features of statistics information could be obtained effectively and accurately. The improved principal component analysis method of relative principal component algorithm ${ }^{[12,13]}$ among multivariate statistics analysis is adopted and according to related literatures ${ }^{[13-16]}$ the basic algorithm procedures are shown as follows.

1) the received multi-frequency ultrasonic signals are divided into $N(N>100)$ samples, and each sample contains $M$ data, including the ultrasonic amplitude, phase, frequency, and flight time. The 
following formula (1) is used to perform standardization on these dada, and after standardization, the data average value and variance are respectively.

$$
\begin{aligned}
& \boldsymbol{X}^{R}=\left[\begin{array}{ccc}
x_{1}(1), x_{2}(1) & \ldots & x_{N}(1) \\
x_{1}(2), x_{2}(2) & \ldots & x_{N}(2) \\
\cdot & \cdot & \cdot \\
\cdot & \cdot & \cdot \\
\cdot & \cdot & \cdot \\
x_{1}(M), x_{2}(M) & \ldots & x_{N}(M)
\end{array}\right]\left[\begin{array}{cccc}
Q_{1} & 0 & \ldots & 0 \\
0 & Q_{2} & \ldots & 0 \\
\cdot & \cdot & . & \cdot \\
\cdot & \cdot & . & \cdot \\
\cdot & \cdot & . & . \\
0 & 0 & \ldots & Q_{N}
\end{array}\right] \\
& =\left[\begin{array}{rrrr}
x_{1}^{R}(1) & x_{2}^{R}(1) & \ldots & x_{N}^{R}(1) \\
x_{1}^{R}(2) & x_{2}^{R}(2) & \ldots & x_{N}^{R}(2) \\
\cdot & \cdot & \cdot & \cdot \\
\cdot & \cdot & \cdot & \cdot \\
\cdot & \cdot & \cdot & \cdot \\
x_{1}^{R}(M) & x_{2}^{R}(M) & \ldots & x_{N}^{R}(M)
\end{array}\right]
\end{aligned}
$$

Where $\boldsymbol{X}^{\boldsymbol{R}}=\boldsymbol{X} \boldsymbol{R}$, and $\boldsymbol{X}^{\boldsymbol{R}}$ is calculated matrix of $\boldsymbol{X}$ after it through the transformation matrix $\boldsymbol{Q}$, and $\boldsymbol{Q}$ is transformation matrix, $Q_{i}(i=1,2, \ldots, N)$ is weight coefficient and its value can be calculated according to the literature $[18,19]$.

2) Calculate the covariance matrix $\boldsymbol{R}_{X}{ }^{R}$ of the relative matrix $\boldsymbol{X}^{R}$,

$$
\boldsymbol{R}_{X^{R}}=\frac{\left(\boldsymbol{X}^{\boldsymbol{R}}\right)^{T} \boldsymbol{X}^{\boldsymbol{R}}}{N-1}
$$

3) Solve eigenvalues $\lambda_{i}$ of the covariance matrix $\boldsymbol{R}_{X}{ }^{R}$ and the corresponding eigenvalues $p_{i}, \lambda_{i}$ is the $i^{\text {th }}$ eigenvalue of $\boldsymbol{R}_{X}{ }^{R}$, which meets

$$
\left|\lambda_{i} \boldsymbol{I}-\boldsymbol{R}_{X^{R}}\right| p_{i}=0
$$

Where $\boldsymbol{I}$ is unit matrix.

4) The relative principal element $j$ is calculated through the contribution ratio and the concrete formula is

$$
\sum_{1}^{j} \lambda_{i}>\sum_{1}^{M} \lambda_{i} * 85 \%
$$

5) Calculate the matrix $\boldsymbol{T}$

$$
\boldsymbol{T}=\boldsymbol{X P}\left(N_{j}\right)=(\boldsymbol{N M})\left(M_{j}\right)
$$

Where the data included in matrix $\boldsymbol{T}$ are the transformation data of main parameters of ultrasonic signals according to the relative principal algorithm, and these data will be processed further based on the complex artificial neural network algorithm.

Complex Artificial Neural Network (CANN) Data Analysis Principle. The CANN has good self-learning ability of conventional artificial neural network and also has adaptive ability of self-weight values adjustment as well as of overcoming the defects of conventional artificial neural network in phase process, and achieves node and weight value ranges transformation from conventional real number field to complex field, and aimed at large data demand of higher-order statistical value algorithm, and the case that the second-order statistical value algorithm cannot optimize the defects contain common zero channels. The CANN algorithm gets complex numbers of the neural weight values, and the principle schematic diagram of the algorithm is shown in Fig.1 below, which is mainly composed of input layer, hidden layer and output layer. 


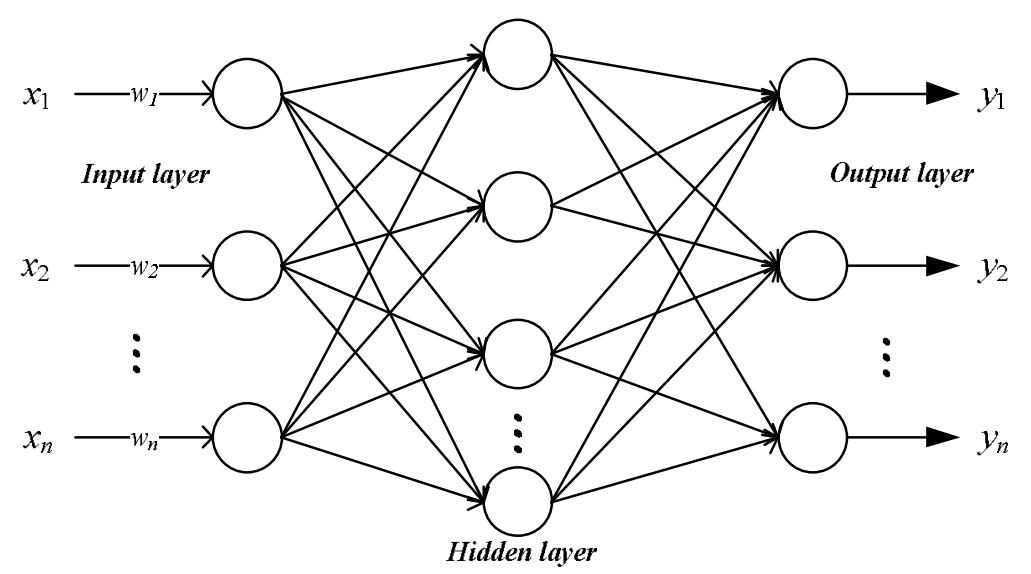

Fig.1 Artificial neural network structure diagram

In Fig. $1, w_{i}$ is connection strength between the neural cells, is called complex weight value $i$. Firstly, the data samples $\boldsymbol{X}=\left[x_{1}, x_{2}, \ldots, x_{n}\right]^{\mathrm{T}}$ in database (the data samples are same with the data obtained from multivariate statistics analysis method) and corresponding fault eigenvalues are set as the given signals of $\boldsymbol{Y}^{*}=\left[y_{1}{ }^{*}, y_{2}{ }^{*}, \ldots, y_{\mathrm{n}}{ }^{*}\right]^{\mathrm{T}}$, and the given signals can be learned adaptively by the artificial neural network, and the corresponding input complex weight values $\left[w_{1}, w_{2}, \ldots, w_{n}\right]$ can be trained.

$$
y_{i}=f\left(x_{\mathrm{i}}\right)=P\left(w_{1} x_{1}+w_{2} x_{2} \ldots+w_{n} x_{n}\right)
$$

When $2 \pi j / k \leq \arg (z) \leq 2 \pi j /(k+1)$, its activation function is defined ${ }^{[20,21]}$

$$
P(z)=e^{\frac{2 \pi j}{k} i}
$$

Where $j \in\{0, k-1\}, i$ is imaginary unit, $z=w_{1} x_{1}+w_{2} x_{2}+\ldots+w_{n} x_{n}, \arg (z)$ is argument of complex $z$.

According to formula (6) and (7), the output variable $y_{i}$ corresponds to the input variable $x_{i}$, namely,

$$
\Delta y_{i}=y_{i}^{*}-y_{i}
$$

According to formula (8), the deviation amount $\Delta y_{i}$ is computed by following formulas:

$$
\begin{aligned}
\Delta w_{i} & =\Delta y_{i} \eta \bar{x}_{i} \\
w_{i}(k+1) & =w_{i}(k)+\Delta w_{i}
\end{aligned}
$$

The formula (9) shows the deviation amount of weight value, among which, $\eta$ is learning rate, $\bar{x}_{i}$ is conjugate complex number of input vector. In formula (10) the $w_{i}(k)$ is current weight value, $w_{i}(k+1)$ is weight value after learning.

\section{Transformer Faults Detection Based on Multivariate Statistics and Complex Artificial Neural Network}

Self-learning based on Artificial Neural Network (ANN). The ANN parameters are selected according to the learning sample ${ }^{[18]}$, and which are selected as each fault eigenvalue of transformer in this paper. Due to different aroused eigenvalue corresponding to each kind of transformer fault, such as low voltage interphase short cut, insulation structure overheating, and tap switch flying arc or spark discharge, and other kinds of transformer faults, etc. The amounts of samples in database are transformer fault eigenvalues, and which have 1200 groups of data as the learning sample. Therefore, the number of input nodes of selected complex artificial neural network in the paper is 20 and the output number is 23 , and the number of nodes in used hidden layer is determined by network self-learning in continual adjustment.

The data analyzed from multivariate statistics is selected as input matrix $X$ of ANN, and the objection is outputting matrix $Y(1200 * 23)$, and the learning rate $\eta=0.1$, and error coefficient is 0.0001 ${ }^{[14,15]}$. During the initialization, each neural branch weight value is unified set as 1 and the self-learning is started according to transformer fault values and the received multi-frequency ultrasonic eigenvalues, each fault eigenvalue trained is correspond to the complex weight value $w_{i}$.

The ANN self-learning flow chart is shown in Fig.2 below. 


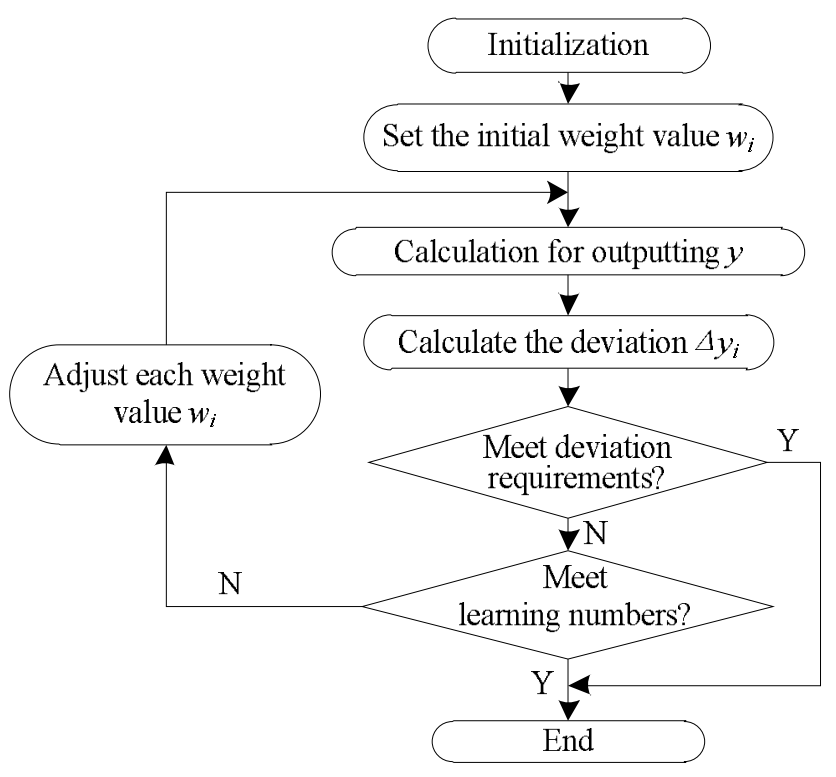

Fig.2 Self-learning flow chart of ANN

The flow chart of self-learning is shown as follows.

Step1: complex weight value, error coefficient and learning numbers initialization, the $w_{i}$ in the thesis is constant 1 , and the error coefficient is 0.0001 and the learning numbers is set as 10000 .

Step2: compute and output $y_{i}$, and utilize $y_{i}=f\left(x_{i}\right)=P\left(w_{1} x_{1}+w_{1} x_{l}+\ldots+w_{n} x_{n}\right)$, and calculate the output variable $y_{i}$ which is correspond to the input variable $x_{i}$, and the activation function is defined when $2 \pi j / k$ $\leq \arg (z) \leq 2 \pi j /(k+1), P(z)=e^{(2 \pi j / k)^{*} i}$, among it, $j \in\{0, k-1\}$, and $i$ is imaginary unit, $\arg (z)$ is amplitude angle of complex $z$, and $z=w_{1} x_{1}+w_{1} x_{1}+\ldots+w_{n} x_{n}$.

Step3: calculate the difference variable $\Delta y_{i}=y_{i}{ }^{*}-y_{i}$.

Step4: according to step3, the difference variable $\Delta y_{i}$ can be calculated to judge whether the errors meet requirements, and the error coefficient is set as 0.0001 in the thesis, if $\Delta y_{i}>0.0001$, then execute the Step5, otherwise, execute the adaptive learning in this step until it is finished.

Step5: judge whether it is reached to preset learning numbers. The learning cycle numbers $n$ is set 10000 in the thesis, if $n>10000$, the learning of this step is finished, otherwise, implement the Step6.

Step6: adjust the complex weight value. According to formula (6) (7), the weight value deviation can be computed, and which is added to previous complex weight value, namely, $\Delta w_{i}=\Delta y_{i} \eta \bar{x}_{i}$, $w_{i}(k+1)=w_{i}(k)+\Delta w_{i}$, among them, $\eta$ is called learning rate, $\bar{x}_{i}$ is conjugate complex number of input vector, $w_{i}(k)$ is current weight value, $w_{i}(k+1)$ is weight value after learning.

Step7: repeat Step1 to Step6, until the learning is finished, then the complex weight value after learning can be obtained.

To Solve the Problems in Transformer Oil. Finally, according to the data matrix $T$ obtained by multivariate statistics analysis discussed in section II. $A$ and the trained complex weight values in section II. $B$, the transformer oil parameters presented in section II. $A$ can be computed, and finally, the obtained eigenvalues by calculation are compared with the eigenvalues in the database, so as to get the name of faults.

\section{Design of Multi-frequency Ultrasound Transformer Detection System}

The Whole System Structure Design. The whole system structure principle is shown in Fig.3, which contains the ultrasonic emission and receiving unit, the multi-frequency ultrasonic sensor and the data analysis system software. 


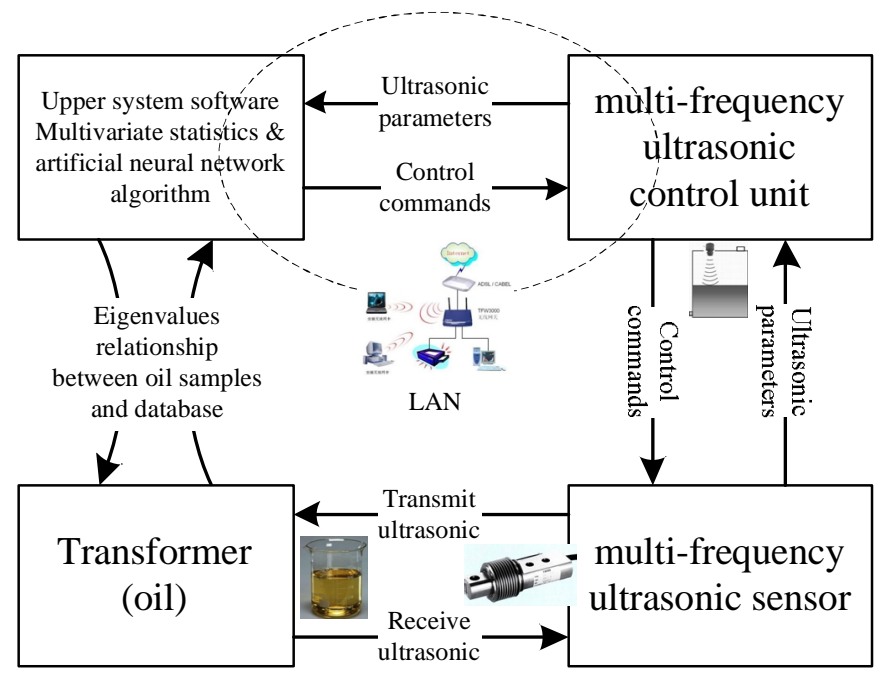

Fig.3 The design of multi-frequency detection system.

In Fig.3, the power interface, the power switch, the Internet interface, the ultrasonic signal output interface and receiving interface are installed on the ultrasonic transmission and receiving control unit. Each main functional part is introduced in details as follows.

The ultrasonic transmission and receiving control unit (UTRCU) includes the interfaces introduced above, and the unit is mainly used to control the multi-frequency ultrasonic signal emission and receiving. In the unit the multi-frequency unit generators are set, and whose internal circuits are connected to ultrasonic signal output interface in UTRCU, and the output interface is connected to the multi-frequency ultrasonic sensor by conductors. The output interface of multi-frequency sensor is connected to the receiving interface of UTRCU, and then the signals in ultrasonic receiving interface are transmitted to the signal processing circuits in UTRCU, and the control circuit is designed based on DSP technique and can accurately finish the calculation from aspect of ultrasonic original signals to the ultrasonic parameters of amplitude, phase and ultrasonic speed. Finally, through the network interface, each ultrasonic parameter is uploaded to the upper system analysis software.

Multi-frequency ultrasonic sensor, in order to ensure the detection precision, which is required to keep the surfaces of all sensors in accordance, thus, it's necessary to utilize the electronic approach to guarantee unified correction of sensors.

The upper data analysis software is mainly adopted to collect the multi-frequency ultrasonic eigenvalues for analysis, so as to establish the correlation between the eigenvalues and oil parameters. The functions of the designed upper system analysis software are achieved based on multivariate statistics analysis technique, such the principal components analysis (PCA) and linear discriminant analysis (LDA), etc.

According to Fig.3, as a whole, the emission control module generates the carrier pulse signal required by the emission of transducers, according to the output signals of frequency control module and power control module, so as to achieve function of transducers transmitting power. The pulse signals output by the emission control module are boosted by the emission driving module, and then it produces output signals with large power, thus drives the transducers to transmit suitable multi-frequency ultrasonic signals.

This part can set a satisfied frequency and power based on the size Pfizer transformer capacity, thus greatly improve the detection efficiency. In the upper system analysis software, the multivariate statistics analysis and complex artificial neutral network analysis algorithms are included, and based on related algorithms, the micro water content, the breakdown voltage, the dielectric loss, the volume resistivity, and the oil interfacial tension and other parameters can be obtained from the transformer oil parameters, and these transformer oil parameters are made comparisons with the transformer fault eigenvalues in the background database, finally we can judge the transformer operation situation and timely find the faults. 
Design of the Emission Part and Receiving Part. (1) Design of the emission part. The design of the emission part in the ultrasonic emission and receiving unit is shown in Fig.4, this part mainly contains the power control module, the emission control module, the frequency control and the emission driving modules.

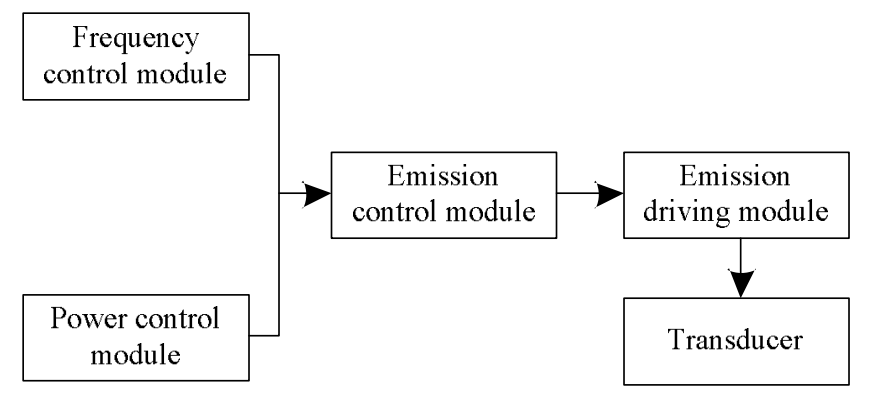

Fig.4 Design of the receiving part

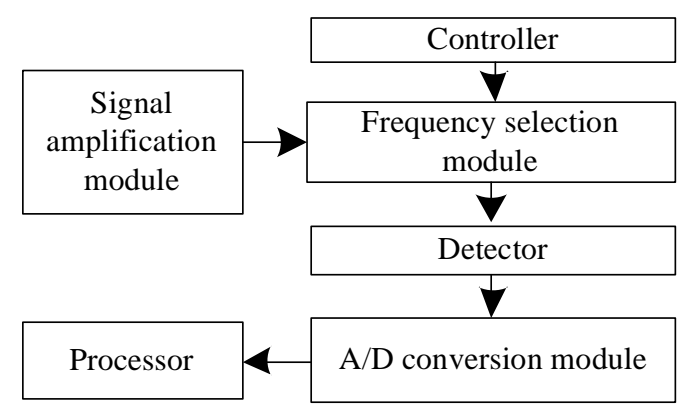

Fig.5 Design of the emission part

Based on Fig.4, the power control module selects satisfied power signal commands based on the transformer types and size of transformer capacity, and the commands are sent to the emission control module for procession; the emission control module produces the carrier pulse signals which are needed by the transducer, and this emission process is based on the output signals from the frequency control module and power control module, the carrier pulse signals are transmitted to the emission driving module, and the frequency determines the carrier frequency, the power determines the pulse width and amplitude, so as to achieve power emission control function on the transducer; the emission driving module can boost the pulse signals from emission control module and then generates output signals with large power, and which can drive the transducer to transmit more suitable ultrasonic signals. This part can set a suitable frequency and power according to the size of transformer capacity and thus greatly improve the detection efficiency.

(2) Design of the receiving part. The receiving part of ultrasonic emission and receiving control unit is shown in Fig.5 above, and which is mainly composed of the signal amplification module, the frequency selection module and the analog digital conversion module (A/D conversion module). According to Fig.5, the signals transmitted from the ultrasonic sensor are amplified by the signal amplification module and then are sent to the frequency selection module, in which the frequency of each signal is selected and a suitable frequency range is determined, and so that the interference signals are filtered, and we can obtain accurate multi-frequency ultrasonic signal analogs and which are converted into digital signals and sent to the microprocessor for further smart analysis.

\section{Experimental Analysis}

The Principle Scheme of the Whole Detective System for Learning Process. Firstly, the detective system generates middle-frequency-ultrasound (MF-ultrasound) signals with frequencies of $f_{1}, f_{2}, \ldots, f_{n}$, and which are transmitted to the oil \&transformer model to obtain the ultrasound parameters and transformer oil parameters, among them, in the former each ultrasound parameters contains the TOF, phases, amplitudes, etc., and the latter contains the temperature, acidity, relative humidity, loss factor, BDV, water content, particles, and DP-value, etc. also they can be divided into two parts, one is the oil parameter, and the other is solid parameter. The ultrasound parameters and the transformer oil parameters are measured by the specific equipment, and then these data are made in fusion to get the inference engines via a series of inferences. Meanwhile, the solid parameters and oil parameters are uploaded to the background database via the communication interface, a multivariate statistics algorithm is applied to build a relationship between the measured data and sample data in database. Finally, the name of fault occurred in transformer oil can be got, and the corresponding measures are took. The learning process of the detective system is shown in Fig.5. 


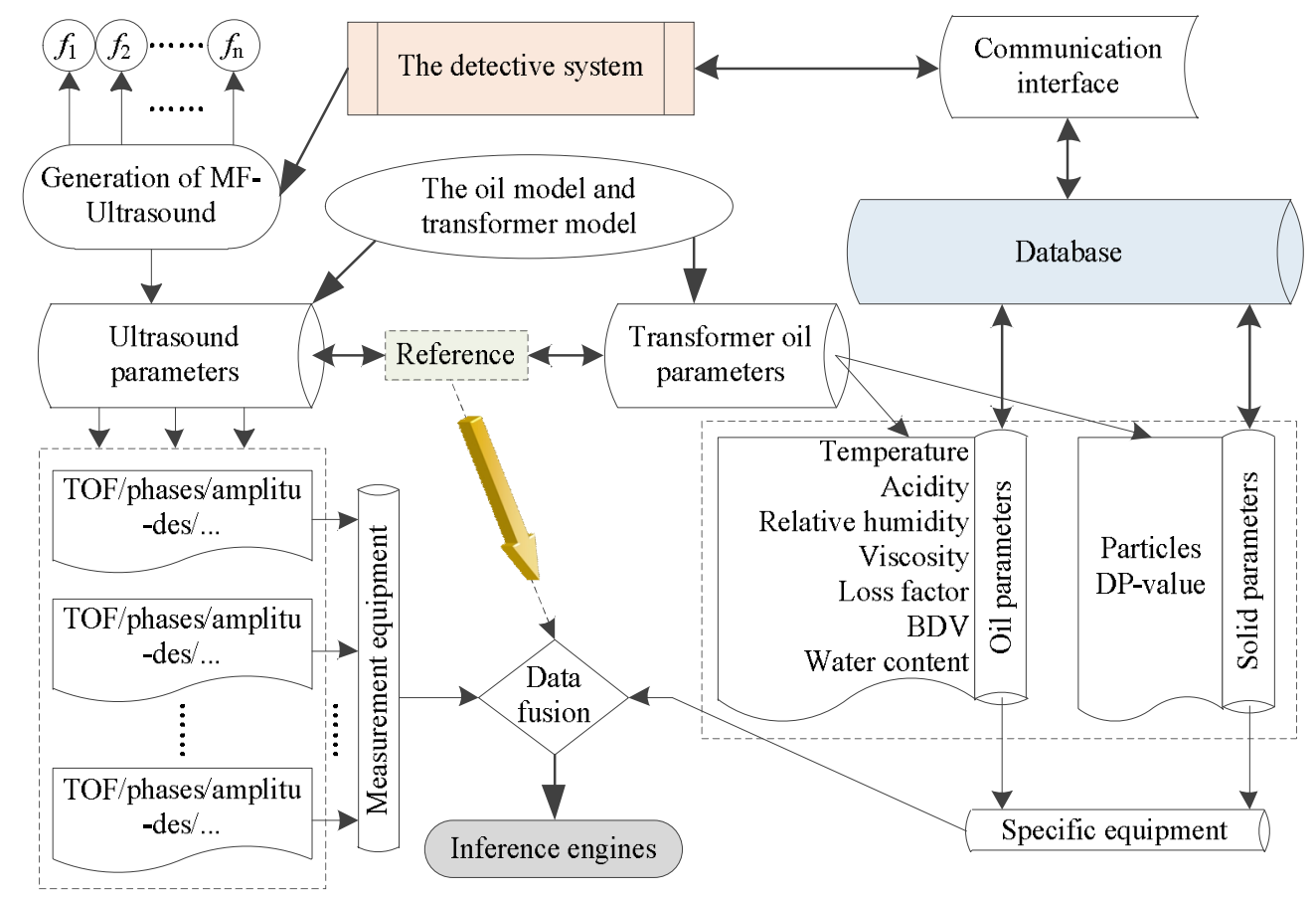

Fig.6 The learning process of the whole detective system

In practical test, the reference values of oil parameters are according to the international standards IEC 60422, and which includes IEC 60814 "Water content (absolute, Karl Fischer-relative, Vaisala)", IEC 60156 "Electrical breakdown voltage (BDV)", IEC 60247 "Dielectrical dissipation factor (DDF/tand/power factor)", IEC 62021 "Acidity (neutralization number)", IEC 60567 "Gas-in-oil-Analysis", IEC 61125 "Sediment and Sludge content", IEC 61125 "Oxidation resistance", IEC 60666 "Inhibitor content (Anti-oxidant additives)" and IEC 60970 "Particles".

In practical test, the concrete connection principle is shown in Fig.7 below.

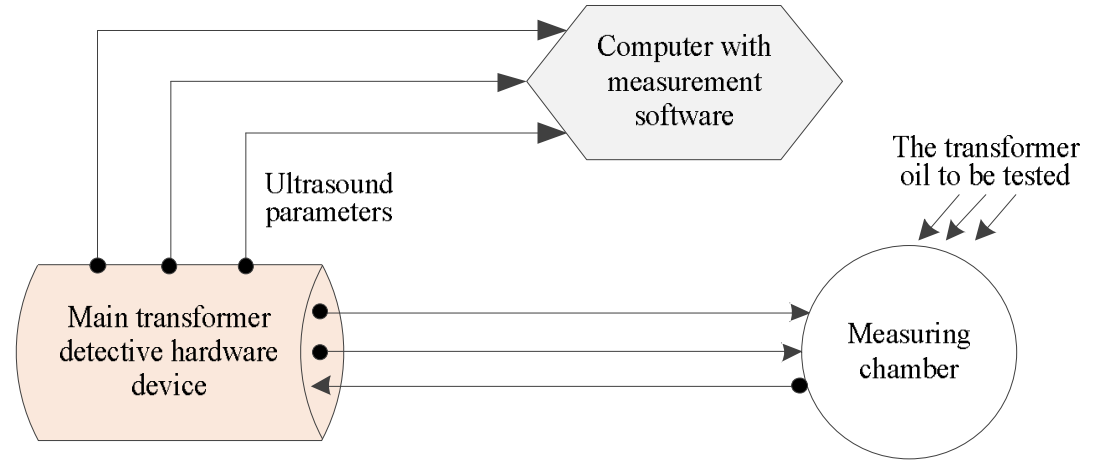

Fig.7 The practical detection connection principle

Set the parameters of water content and breakdown voltage as an example, its testing interfaces are shown in Fig.8.

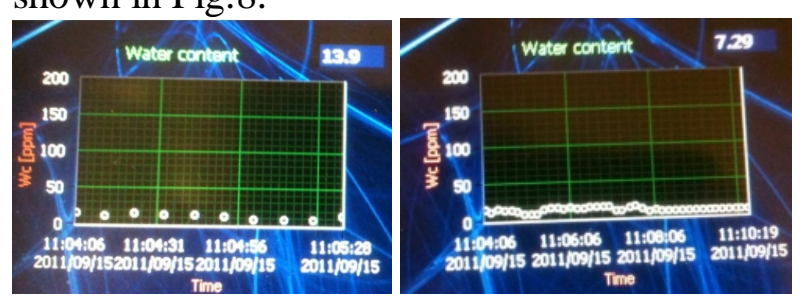

(a) Water content
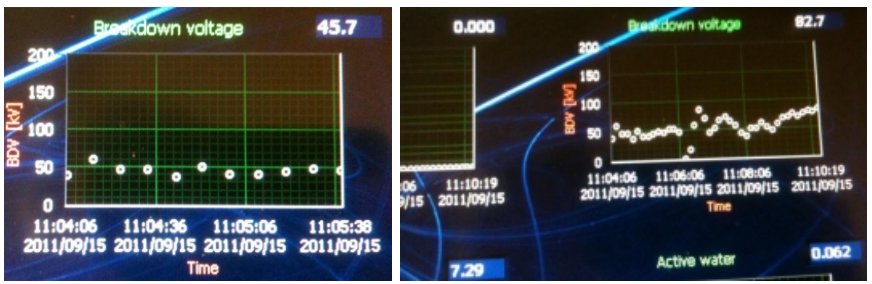

(b) Breakdown voltage

Fig.8 The testing interfaces of water content and breakdown voltage 
The water content of transformer oil can be calculated according to the following formula ${ }^{[22]}$,

$$
p p m=R H \times 10^{(A+B / T)}
$$

Where the $R H$ is relative humidity, which is obtained from the water content in practical oil, for the sensor $P-14$, utilize the least square method, $A=7.3659, B=1661.5$, then we can get the value of $p p m$.

The breakdown voltage is an important index to evaluate the electric strength of transformer oil, and is also dimension to balance the withstand voltage ability of transformer oil inside transformer. Substantially, the breakdown voltage reflects the existence of the moisture, impurities, and conductive particles and the severity of them on insulation performance of transformer oil. The standard recommended values of breakdown voltage in transformer oil are shown in Tab. 1 below ${ }^{[23]}$.

Tab.1 The standard recommended values of breakdown voltage in transformer oil

\begin{tabular}{cccc}
\hline \multirow{2}{*}{ Item } & $\begin{array}{c}\text { Voltage level of equipment } \\
/ \mathrm{kV}\end{array}$ & \multicolumn{2}{c}{ Quality indicators } \\
\cline { 2 - 4 } & $750 \sim 1000$ & $\begin{array}{c}\text { Oil before putting into } \\
\text { operation }\end{array}$ & Oil in operation \\
\hline \multirow{2}{*}{ Breakdown voltage } & 500 & $\geq 70$ & $\geq 60$ \\
$/ \mathrm{kV}$ & 330 & $\geq 60$ & $\geq 50$ \\
& $66 \sim 220$ & $\geq 50$ & $\geq 45$ \\
& 35 et seq & $\geq 40$ & $\geq 35$ \\
& & $\geq 35$ & $\geq 30$ \\
\hline
\end{tabular}

It's seen from Fig. 8(a) and (b) that the transformer-oil water content is tested between 10 20VVc [also uses unit of ppm, and $1 \mathrm{ppm}=1 \mathrm{mg} / \mathrm{kg}=1 \mathrm{mg} / \mathrm{L}$ ] and the breakdown voltage $40 \sim 60 \mathrm{kV}$ and average value closes to $50 \mathrm{kV}$, and the active water is about $0.05 \mathrm{Avv}$. According to the measurement parameters the state of transformer-oil can be made a deep analysis, and we can concluded that 1) the ppm of water content is about 10 20 mg/L, which is met with the IEC 60814 standard "Determination of water by automatic coulometric Karl Fischer titration") and 2) the breakdown voltage (BDV) is about $40 \sim 60 \mathrm{kV}$ (the insulation voltage of equipment is about $66 \sim 220 \mathrm{kV}$, and the tested oil has been put into operation for several years), which is also met with the IEC 60156 standard ("Determination of the breakdown voltage at power frequency -Test method"). Thus we can judge the health state of current transformer oil easily based on the oil parameters, and the transformer faults can be got conveniently.

\section{Conclusions}

In this paper, based on multi-frequency ultrasonic technique (MFUT), the multivariate statistics and complex artificial neural network (CANN) data analysis algorithms were adopted to implement detection on transformer and further judge the fault types. It's mainly aimed at the principal component analysis (PCA) in multivariate statistics and the CANN, and the theoretical analysis was made, and its inferential process and the self-learning realization process were introduced. According to the proposed multivariate statistics analysis technique and CANN data analysis technique, and the whole system analysis software with a strong database was established and the database contained various types of oil sample. Based on the system analysis software, the relationship between theses eigenvalues and oil sample parameters were built according to the CANN smart algorithm. Thus, aimed transformer fault detection, it's concluded from the thesis that

1) the designed multi-frequency ultrasonic transformer detection system can utilize the multi-frequency ultrasonic technique to detect the transformer oil parameters online and in the system software, the detected transformer oil parameters can be made analysis based on the multivariate statistics analysis algorithm, during the detection process, the ultrasonic signal eigenvalue information is extracted and through modeling, the relationship model between the transformer oil situation and ultrasonic eigenvalues is established, further the transformer operation state can be judged based on the relationship model. 
2) the designed transformer fault detection system based on multi-frequency ultrasonic technique can improve the detection ability of transformers in power corporations, and can help them timely understand the whole operation situation of transformers, and the detection data can truly reflect transformers' actual operation conditions, and is able to guarantee safe and stable operation of transformers, and which solves the problems of conventional transformer detection devices, for example, the complicated detection procedures, failed in online detection, etc., thus can improve the detection sensitivity and reduce the detection cost..

\section{Acknowledgements}

This work was gratefully acknowledge Dr. Thomas Fritsch \& Dr. Miroslaw Wrobel from Yucoya Energy Safety GmbH, Würzburg, they gave the hardware design and upper software development of the product TranSCoM Online, also gratefully acknowledge the support of the Science and Technology Project of China Southern Power Grid (GZGD20150301240091) cooperated by the Electric Power Research Institute of Guangzhou Power Supply Bureau Co., Ltd and Huatian Power Technology Co., Ltd.

\section{References}

[1] Q. Yu, Online Monitoring Expert System of Transformer Oil Chromatography. Master's Thesis, Shanghai Jiao Tong University, Shanghai, China, 2007.

[2] J.G. Lai, H. Zhou, "Experimental Research on Parallel Operation for Multiple Micro-inverters," The Journal of New Industrialization, vol. 4, no. 5, pp. 60-68+76, 2014.

[3] S.G. Huang, X.Q. Zhu, S.Z. Peng, B. Qin, "Control Strategy of VSC-HVDC System Supplying Power for Isolated Passive Load," The Journal of New Industrialization, vol. 5, no. 3, pp. 54-60, 2015.

[4] X.H. Chen, Q. Xia, "Using Chromatographic Analysis of Transformer Failures and Their Ideas Judge Construction," Chinese High-tech Enterprises, vol. 36, no. 12, pp. 76-77, 2014.

[5] W.F. Li, Power Transformer Fault Line Detection Based on Infrared Images. Master's Thesis, Anhui University of Science \& Technology, Hefei, China, 2014.

[6] Y.M. Ren, "The Use of Infrared Temperature Measurement Technology for Power Transformer Fault Diagnosis," Management and Technology of Small and Medium Sized Enterprises, vol. 21, no. 2, pp. 205-206, 2015.

[7] H.J. Yin, The Design and Development of Online Monitoring System for Power Transformer Based on Infrared Temperature Measurement Technology. Master's Thesis, Nanjing University of Science \& Technology, Nanjing, China, 2010.

[8] Q.L. Zhang, Fault Diagnosis Technology of Transformer Based on Gas Analysis and Partial Discharge Detection in Oil. Master's Thesis, Nanjing University of Science \& Technology, Nanjing, China, 2014.

[9] W.N. Xing, Research and Design of Partial Discharge Detection in Power Transformers. Master's Thesis, Agricultural University of Hebei Province, Tianjin, China, 2014.

[10]C.C. Meng, Research on Fault Detection and Application Based on Multi-vitiate Statistical Analysis. Master's Thesis, China Jiliang University, Hangzhou, China, 2013.

[11]M.L. Liu, Fault Diagnosis Method Based on Multi-vitiate Statistical Analysis. Master's Thesis, Nanjing University of Science \& Technology, Nanjing, China, 2013. 
[12]L. Xi, X.S. Zhang, L.F. Cheng, T. Yu, J. Tang, "Research on Multi-agent Simulation Platform for AGC Based on JADE," The Journal of New Industrialization, vol. 4, no. 11, pp. 5-17, 2014.

[13]Z.L. Wu, Fault Diagnosis Method of Power Transformer. Master's Thesis, North China Electric Power University, Baoding, China, 2013.

[14]Cherry G, S.J. Qin, "Multiblock Principal Component Analysis Based on A Combined Index for Semiconductor Fault Detection and Diagnosis," Institute for Electrical and Electronic Engineers Transactions on Semiconductor Manufacturing, vol. 19, no. 2, pp. 159-172, 2006.

[15]Z. Qian, W.S. Gao, Y. Shang, Z. Yan, "Application of Data Reliability Analysis and BP Neural Network for Transformer Fault Diagnosis," High Voltage Engineering, vol. 25, no. 1, pp. 13-15, 1999.

[16]F.F. Zhou, Fault Diagnosis of Power Transformer Based on Neural Network and Evidence Theory. Master's Thesis, Xihua University, Chengdu, China, 2009.

[17]W. Xu, D. Wang, Z. Zhou, H. Chen, "Fault Diagnosis of Power Transformers Application of Fuzzy Set Theory Expert Systems and Artificial Neural Networks," IEEE Proc.Sci. Meas. Technol, vol. 144, no. 1, pp. 39-44, 1997.

[18] Y.S. Qi, P. Wang, X.J. Gao, "On-line Monitoring of Fed-batch Penicillin Cultivation Using Time-varying and Multivariate Statistical Analysis," In: Proceedings of the Conference on Bioinformatics and Biomedical Engineering, Chengdu, China, 18-20 June, 2010, pp. 1-6.

[19] Vanegas O., Mizuno Y., Naito K., Kamiya T., "Diagnosis of Oil-insulated Power Apparatus by Using Neural Network Simulation," IEEE Trans . Diele. and Elect. Insul, vol. 4, no. 3, pp. 290-299, 1997.

[20]J. Hu, Relative Principal Component Analysis Theory and Its Application. Master's Thesis, Henan University, Kaifeng, China, 2008.

[21]W.F. Lv, M. Lin, B.X. Hua, "The Most Robust Design of Digital Logic based on the Plural Artificial Neural Network," Journal of Circuits and Systems, vol. 13, no. 3, pp. 290-299, 1997.

[22]J.D. Tang, "Design of Implementation of Micro-water Transmitter of Transformer Oil based on Modus RTU,” Electronic Measurement Technology, vol. 36, no. 5, pp. 111-118, 2013.

[23]M. Gao, Study on Insulation Characteristics of Transformer Oil at Low Temperatures. Master's Thesis, China Electric Power Research Institute, Beijing, China, 2011. 